\section{VSIMPL: A program to estimate the number of interpretable factors}

\section{THOMAS ROCKLIN and WILLIAM REVELLE Northwestern University, Evanston, Mlinois 60201}

A fundamental question in psychological measurement deals with the number of factors necessary to summarize a domain of items or tests. Having developed a pool of items or administered a battery of tests, one must decide between a few broad and many narrow factors. In making this decision an interpretable solution is sought. In order for a factor solution to be theoretically useful, the meaning of each factor must be interpretable in terms of the variables that load on it. The most interpretable factors are those on which some items have high loadings and others have low (ideally zero) loadings. A solution is most interpretable when each item has a high loading on one and only one factor. This is especially useful in scale construction, since it avoids the problems associated with scoring items on more than one scale.

In practice, psychologists informally apply these principles of simple structure when considering factor pattern matrices. They tend to interpret solutions by listing each salient item for a given factor and ignoring small, although nonzero, loadings. This is one way of determining the number of factors to extract; more and more factors are tried until the solution becomes theoretically uninterpretable. This method, however, is so subjective that it can be expected to lead to differences in the number of factors extracted more as a function of the complexity of the factor analyst than as a function of the complexity of the data.

Another solution is to apply one of the many psychometric rules of thumb. One might plot the successive eigen values and try to apply the scree test (Cattell, 1966) or simply extract factors until the eigen values fall below one (Kaiser, 1960). Another psychometric approach compares the observed eigen values with those expected from random data (Montanelli \& Humphreys, 1976).

A final method is to test the statistical adequacy of the solution. For example, one might test the hypothesis that the residual correlation matrix does not differ from zero (e.g., Joreskog \& van Thillo, Note 1). This approach ignores the question of whether the factors extracted are likely to be interpretable or can be rotated to simple structure. Further, it has the distinct problem of extracting more factors as the number of subjects increases. While each of these rules works well for some data sets, there are other data sets in which each will fail (Revelle \& Rocklin, 1979; Hakstian, Rogers, \& Cattell, Note 2).

The work described in this paper was supported in part by Grant MH 29209 from the National Institute of Mental Health.
Algorithm. Revelle and Rocklin (1979) have recently presented a simple method of estimating the number of factors to extract from a correlation matrix. This method (called very simple structure, or VSS) is simple and incorporates notions from each of the major approaches noted above. VSS asks how well the observed correla. tion matrix may be reproduced from a factor matrix that has been distorted to the simple structure we tend to interpret. Applying VSS involves the following steps.

(1) Where $\mathrm{k}$ is the number of factors extracted, find factor solutions for values of $k$ ranging from slightly greater than to slightly less than the theoretically expected value of $k$. These may be found by any preferred extraction algorithm.

(2) Rotate the solution to any one of a variety of simple structure criteria including varimax, quartimax, or some oblique transformation. Call the rotated factor pattern matrix $\mathrm{F}_{\mathrm{k}}$.

(3) Apply the VSS criterion: (a) For a VSS solution of complexity $v$, replace the $k-v$ smallest elements of each row of $F$ with zeroes. Call this matrix $S_{\mathrm{vk}}$. The subscripts are $\mathrm{v}$ for the complexity of each item and $\mathrm{k}$ for the number of factors. This step operationalizes the informal way in which we interpret factor solutions. Unless $\mathrm{F}$ has a simple structure of complexity $\mathrm{v}, \mathrm{S}$ will be a simplified or degraded form of $F$. (b) As a way of indexing the fit of a simple structure model of complexity $v$ to the data, consider how well the matrix

$$
\mathrm{R}_{\mathrm{v}}=\mathrm{S}_{\mathrm{vk}} \Phi \mathrm{S}_{\mathrm{vk}}
$$

(where $\Phi$ is the factor intercorrelation matrix) corresponds to the original correlation matrix, R. To do this, find the residual matrix:

$$
\bar{R}_{\mathrm{V}}=\mathrm{R}-\mathrm{R}^{*}{ }_{\mathrm{v}}=\mathrm{R}-\mathrm{S}_{\mathrm{vk}} \Phi \mathrm{S}_{\mathrm{vk}}^{\prime}
$$

(c) To index the fit of the residual matrix to the original matrix, compute:

$$
\mathrm{VSS}_{\mathrm{vk}}=1-\mathrm{MS}_{\overline{\mathrm{r}}} / \mathrm{MS}_{\mathrm{r}} \text {, }
$$

which is 1 minus the ratio of the mean square residual correlation to the mean square original correlation. The degrees of freedom for these mean squares are the number of correlations estimated minus the number of free parameters in $S_{\mathrm{vk}}$. The mean squares are found for the off-diagonal elements in the original and residual correlation matrices.

(4) The optimal number of factors to extract is given by the value of $k$ that maximizes VSS $_{v k}$ for the value of $\mathrm{v}$ thought appropriate. Usually (especially in scale construction), a complexity of one is thought to be appropriate, but the investigator may easily have theoretical reasons for believing that some higher value of 
$\mathbf{v}$ is more appropriate for a particular data set (i.e., a bifactor model may be represented with $v=2$ ). It should be noted that for a given $k$, higher values of $v$ will normally lead to higher goodness-of-fit indices.

Why does the VSS criterion work? Imagine a correlation matrix that is composed of three clusters of variables. Within each cluster, the variables are highly correlated, while between clusters the correlations are negligible. Clearly, VSS will increase from $k=1$ to $k=3$, but why should it decrease thereafter? By assigning some items to the fourth factor, we are saying that they do not correlate with items in the first three factors, which is untrue, given the three-factor structure we have described.

Accuracy of the Algorithm. Revelle and Rocklin (1979) compare this method with several others on both simulated and real data. The simulated data share the characteristics of much questionnaire data: low communalities (about .3), items of complexity of one, and probably not as many subjects as one would like (n ranging from 50 to 400 for 24 variables). VSS did very well, correctly identifying the number of factors in 30 out of 32 simulations. The VSS solutions for the real data sets made good theoretical sense.

Program Operation. A computer program has been written in FORTRAN IV that applies the VSS criterion. The program, VSIMPL, operates in one of three modes. In Mode 1, a correlation matrix and a series of factor pattern matrices (alternating with interfactor correlation matrices in the oblique case) are the input to the program. In Mode 2, this information is read from an SPSS output file. In Mode 3, which will only work at CDC installations operating under SCOPE, VSIMPL submits an SPSS job to do the factor analysis and then proceeds as in Mode 2. Several features of VSIMPL are nonstandard, but in each case, internal documentation points out the feature and suggests ways for a local programmer to adapt VSIMPL.

Output. The results reported by VSIMPL include residual matrices and item goodness-of-fit statistics, along with the overall goodness-of-fit estimate for a given $\mathbf{v}$ and $\mathrm{k}$. A final summary of the goodness of fit for all values of $v$ and $k$ requested by the user is provided. Additionally, if CALCOMP plotting subroutines are available, a plot of the goodness of fit as a function of $\mathrm{v}$ and $\mathrm{k}$ can be requested.

Restrictions. The current compilation of VSIMPL allows for 50 variables and an unlimited number of subjects. By redimensioning arrays, this can be increased until core space is exhausted. VSIMPL requires $62 \mathrm{~K}$ (octal) words of memory on a CDC 6600 for a 50-variable problem.

Availability. The source deck and test data, along with sample output and complete user documentation, are available from the second author for $\$ 10$ to cover costs.

\section{REFERENCE NOTES}

1. Joreskog, K. G., \& van Thillo, M. New rapid algorithms for factor analysis by unweighted least squares, generalized least squares, and maximum likelihood (ETS RM 71-5). Princeton, N.J: Educational Testing Service, 1971.

2. Hakstian, A. R., Rogers, W. T., \& Cattell, R. B. The behavior of number-of-factors rules with simulated data. Paper presented at the annual meeting of the Society of Multivariate Experimental Psychology, Los Angeles, November 1979.

\section{REFERENCES}

CAttell, R. B. The scree test for the number of factors. Multivariate Behavioral Research, 1966, 1, 245-276.

KAISER, H. F. The application of electronic computers to factor analysis. Educational and Psychological Measurement, 1960 , 20, 141-151.

Montanelli, R. G., \& Humphreys, L. G. Latent roots of random data correlation matrices with squared multiple correlations in the diagonal: A Monte Carlo study. Psychometrika, $1976,41,341-348$.

Revelle, W., \& Rocklin, T. Very simple structure: An alternative procedure for estimating the optimal number of interpretable factors. Multivariate Behavioral Research, 1979, 14, 403-414.

(Accepted for publication January 8, 1980.) 